\title{
Entendiendo el Acuerdo de Medidas Sanitarias y Fitosanitarias (MSF) de la Organización Mundial del Comercio (OMC) ${ }^{1}$
}

Edward A. Evans ${ }^{2}$

\section{Introducción}

Esta publicación es parte de una serie que trata de políticas de comercio. La liberalización del comercio internacional y la globalización general de la economía mundial en las últimas dos décadas ha significado que agricultores, comerciantes y grupos en la comunidad se familiarizen con políticas de comercio y su terminología para poder beneficiarse de la globalización y para minimizar costos.

Consequentemente, el objetivo de esta serie de publicaciones es ayudar a la gente a entender los tópicos del comercio mundial que benefician o dan problemas.

Esta publicación, la primera de una serie, discute el término "Acuerdo de MSF de la OMC" (Acuerdo de Medidas Sanitarias y Fitosanitarias de la Organización Mundial de Comercio).

\section{¿Qué es el Acuerdo de Medidas Sanitarias y Fitosanitarias de la OMC?}

OMC es la abreviatura de la Organización Mundial del Comercio. Esta es la única organización internacional que regula el comercio entre países. La parte central de la OMC es un conjunto de acuerdos negociados, firmados y ratificados por los gobiernos de la mayoría de países que comercian en el mundo. Estos acuerdos son las bases legales del comercio internacional. Ellos estimulan a los gobiernos para que mantengan sus políticas de comercio dentro de los lmites de los acuerdos para el beneficio de todos (http://www.wto.org/indexsp.htm).

Las Medidas Sanitarias (seguridad humana y animal) y Fitosanitarias (seguridad de plantas) [Acuerdo de las MSF] es uno de los acuerdos que en 1994 estrablecieron la OMC, la que reemplaza el Acuerdo General sobre Aranceles Aduaneros y Comercio (GATT). Las regulaciones (Apedice A de

1. Este documento, FE530, es uno de una serie de publicaciones del Departamento de Food and Resource Economics, Servicio de Extensión Cooperativa de la Florida, Instituto de Alimentos y Ciencias Agrícolas, Universidad de la Florida. (UF/IUFAS). Fecha de primera publicación: February 7, 2005. Visite nuestro sitio web EDIS en 〈http://edis.ifas.ufl.edu>.

2. Edward A. Evans, Assistant Professor, Department of Food and Resource Economics, Tropical Research and Education Center, Homestead, FL, Florida Cooperative Extension Service, Institute of Food and Agricultural Sciences, University of Florida, Gainesville, FL.

El Instituto de Alimentos y Ciencias Agrícolas es un empleador que opera bajo Acción Afirmativa y provee Oportunidades Igualitarias, autorizado a proveer investigación, información educativa y otros servicios, únicamente a los individuos e instituciones que operan sin discriminación alguna con relación al credo, color, religión, edad, incapacidad, sexo, orientación sexual, estado civil, nacionalidad, opinion política o afiliaciones. Para más información sobre como obtener otras publicaciones de extensión, comuníquese con la oficina de Servicio de Extensión de su condado. Servicio de Extensión de la Florida / Instituto de Alimentos y Ciencias Agrícolas / Universidad de la Florida / Larry Arrington, Decano Interino. 
las MSF de la OMC de 1994.

http://www.wto.org/spanish/tratop_s/sps_s/ spsagr_s.htm) que están dentro del acuerdo de las MSF de la OMC son medidas aplicadas para:

- la protección de la salud y vida de animales y plantas dentro de un territorio contra riesgos que se presenten por la entrada o diseminación de pestes, enfermedades, organismos portadores de enfermedades $\mathrm{u}$ organismos que causen enfermedades.

- la proteción de la vida y la salud de personas y animales dentro de un territorio contra los riesgos que presenten aditivos, contaminantes, toxinas $u$ organismos que causen enfermedades que vienen en los productos alimenticios, las bebidas o los piensos.

- la protección de la vida y la salud humana dentro de un territorio contra riesgos que se presenten debido a enfermedades transmitidas por animales, plantas o productos derivados de ellos, o por la entrada, establecimiento o propagación de pestes.

- prevenir o limitar riesgos o daños dentro de un territorio debido a la entrada, establecimiento o diseminación de pestes.

\section{¿Cuál es la Razón Económica para que los Gobiernos Intervengan?}

La razón para que los gobiernos intervengan en el establecimiento de polítacas y la implementación de medidas que prevengan o controlen la introducción y propagación de pestes y enfermedades se basa en el concepto económico de servicios (bienes) públicos y en el de externalidades. Como se define aquí, servicio público es algo que se da y que es virtualmente imposible prevenir que cualquiera lo use y el hecho que una persona lo use no limita de manera alguna que otra persona también lo use. Como nadie puede ser propietario de tal servicio, si se produjera, su producción no sería suficiente. Por lo tanto es necesario que el gobierno asuma la responsabilidad de producirlo y recupere los gastos a través de impuestos. Un ejemplo de este tipo de servicio público son las medidas que previenen o controlan la diseminación de pestes y enfermedades invasivas. El concepto de servicio público puede extenderse fácilmente hacia el de servico público mundial cuando hay cooperación entre los gobiernos de muchos países que toman acción para reducir, por ejemplo, la treta de pestes y enfermedades invasivas.

Una externalidad se presenta cuando la acción o inacción de una parte afecta a otra de una manera positiva o negativa sin que la parte responsable por la acción o inacción sea recompensada cuando el impacto es positivo o sea penalizada cuando el impacto es negativo. Por ejemplo, una externalidad negativa puede presentarse cuando bienes importados llegan acompañados de pestes o enfermedades invasivas, las cuales pueden reducir la producción o aumentar los costos de producción. Generalmente el país exportador no se hace cargo del costo de los daños que el país importador sufre. En semejantes casos, cuando los mecanismos del mercado por si solos fallan en prevenir o corregir las externalidades negativas, los gobiernos están justificados en proveer regulaciones (servicios públicos) que previenen la entrada o reducen el riesgo de la treta.

La necesidad de un gobierno de proteger a sus ciudadanos y el medio ambiente contra externalidades importadas (como pestes y enfermedades invasivas) es reconocida y aceptada por el acuerdo de la OMC (discutido en seguida), que promueve el comercio entre los países. Veamos por ejemplo dos escenarios. En el primer escenario, regulaciones impuestas por el gobierno para proteger recursos humanos y el medio ambiente contra externalidades negativas (por ejemplo la treta de organismos dañinos) a través de barreras contra el comercio pueden beneficiar al país. En el segundo escenario, regulaciones impuestas con el sólo propósito de proteger a productores domésticos contra la competencia internacional pueden ocasionar daños al país. El primer escenario es aceptable para la OMC, pero no el segundo. Esta naturaleza dual de las MSF (por un lado el potencial genuino de proteger y por el otro el potencial de ser usado como herramienta econmica) ha hecho que su implementación sea muy litigiosa y ha señalado la necesidad de firmar acuerdos de MSF entre gobiernos. 


\section{¿Por qué el Acuerdo de las MSF de la OMC Promueve el Libre Comercio?}

El objetivo principal del Acuerdo de las MSF de la OMC es promover el libre comercio. En principio, un país puede incrementar su ingreso nacional real utilizando eficientemente sus recursos limitados y comerciando, lo que significa que los consumidores pueden disfrutar de niveles más altos de satisfacción y los productores pueden vender sus productos en mercados más amplios. En general, se espera que la economía global en su totalidad se beneficie. Sin embargo, cuando el comercio produce externalidades negativas o costos ocultos (como la importación de pestes y enfermedades) la aceptación de esta premisa general no es clara. Las ganacias del comercio ya no son ciertas.

\section{¿Cuál es el Génesis del Acuerdo de las MSF de la OMC?}

La decisión de negociar un acuerdo separado sobre la aplicación de las Medidas Sanitarias y Fitosanitarias durante el tratado multilateral de comercio de la Ronda de Uruguay GATT 1986-1994 fue el punto de partida en el desarrollo de reglas para tratados multilaterales de comercio y dió [rp,omemcoa a asuntos relacionados al comercio agrícola y al riesgo de importar pestes, enfermedades invasivas y enfermedades transmitidas a través de alimentos. Aunque se reconoció que algunas de las MSF tenían el potencial de impedir el comercio y se las consideró importantes en rondas anteriores de la GATT, ellas fueron relegadas para ser incluidas como parte de otros acuerdos y como excepción de las provisiones principales que promueven el comercio (MSF se encuentran en los artículos originales del GATT, principalmente el artículo XX "Excepciones Generales" y después en la Ronda de Tokio de 1979 en los Acuerdos sobre Obstáculos Técnicos al Comercio, un acuerdo multilateral conocido como Código Modelo).

El impulso para negociar acuerdos separados de las MSF y para tratar asuntos de cuarentena pueden ser atribuidos en general a una integración más profunda de la agricultura en el sistema internacional de comercio (mercados abiertos y comercio libre) y en particular a la decisión de castigar el uso de barreras no arancelarias (cuotas, subsidios, licencias). Muchos países, incluyendo los Estados Unidos, temen que con la reducción en el uso y nivel de estas medidas algunos países importadores empiecen a usar barreras técnicas de comercio (especialmente las MSF) para proteger su comunidad agraria. Consequentemente, el intento del acuerdo fue asegurar que cuando las MSF entren en efecto, ellas se aplicarían solamente para dar seguridad a los comestibles y a la salud de plantes y animales y no para restringir indebidamente a otros países el acceso al mercado (James and Anderson, 1998; Roberts, 1998).

\section{¿Cuáles son las Principales Disposiciones del Acuerdo de MSF?}

Para alcanzar su objetivo, los acuerdos de MSF contienen un grupo de disposiciones substantivas y de procedimiento. Las disposiciones substantivas están diseñadas para proteger la vida y salud humana, de animales y plantas y al mismo tiempo prevenir barreras injustificadas contra el comerico. Las disposiciones de procedimiento crean un marco para mejorar la comunicación entre miembros en lo que se refiere a cambios propuestos a las MSF y a la solución de disputas.

Los acuerdos de MSF de la OMC crean un marco para la protección de fronteras y medidas de erradicación mientras facilitan un comercio más libre. El acuerdo se basa en los siguientes cinco principios generales:

1. Armonización: promueve la adopción de medidas que se ajusten a normasinternacionales, guas y/o recomendaciones de agencias internacionales.

2. Equivalencia: reconocimiento mutuo de medidas diferentes pero equivalentes para alcanzar normas internacionales.

3. No discriminatorio: tratando las importaciones como si fueran producción doméstica.

4. Transparencia: notificando a los socios en comercio de cambios en sus MSF, especialmente cuando ellas difieran de las normas internacionales. 


\section{Regionalización: permite la exportación} continua desde áreas limpias (libre de enfermedades) en los países afectados.

El acuerdo reafirma la libertad de cada país de escoger su nivel apropiado de protección contra pestes y patógenos importados. Sin embargo, cuando estas medidas no se apegan a las normas internacionales, el país importador tiene que investigar cientficamente por qué se necesitan las medidas y cómo ellas controlan el riesgo.

Las disposiciones más importantes se encuentran en el Artculo 5, el que incluye:

- Artículo 5.1 requiere que miembros (cuando sea posible y apropiado) basen sus $M S F$ metodologas de evaluación de riesgos desarrollados bajo los auspicios de la organizición internacional apropiada y relevante.

- Artículo 5.2 estipula que los países deben considerar costos directamente relacionados al riesgo (por ejemplo pérdidas potenciales de producción o costos de control o eradicación), tanto cuando calculen como cuando manejen los riesgos a través de una MSF escogida para proteger la salud de plantas o animales.

- Artículo 5.5 estipula que cada miembro evitará distinciones arbitrarias o injustificadas en los niveles de protección que considere apropiadas (si estos niveles resultaran en restricciones disimuladas contra el comercio internacional) para lograr un objetivo consistente en la aplicación de las MSF.

- Artículo 6 requiere que los protocolos de importación se basen en una evaluación de riesgos para valorar los reclamos de países exportadores de que ciertas regiones están libres de enfermedades o pestes o que la prevalencia de pestes y enfermedades en cuarentena es baja.

\section{¿Cómo se ha Comportado el Acuerdo hasta ahora?}

Todavía no hay un veredicto sobre el comportamiento total del acuerdo de MSF de la
OMC. Por un lado, el acuerdo ha promovido exitosamente el comercio internacional (el objetivo principal del acuerdo) juzgando por el nmero de disputas que han sido solucionadas. Por otro lado, también ha incrementado el riesgo de invasion biológica (como pestes y enfermedades extranjeras que entran al país). $\mathrm{Al}$ restringir las medidas sanitarias y fitosanitarias de un país, el acuerdo ha debilitado la protección nacional contra invasiones biológicas (McNeely, 1999). Esto sucede cuando la preocupación mundial por el medio ambiente está creciendo más rápidamente que el desarrollo de technolgías de control aprobadas (FAO, 2001). La diseminación mundial de pestes y enfermedaes se ha incrementado significativamente así como el costo de su control (Figura 1). Por ejemplo, los gastos del Departamento de Agricultura de los Estados Unidos (USDA) en los programas de erradicación de emergencia han pasado de aproximadamente US $\$ 10$ millones por año a comienzos de 1990 a US $\$ 334$ millones por año en el 2001 (USDA Briefing Room, 2003).

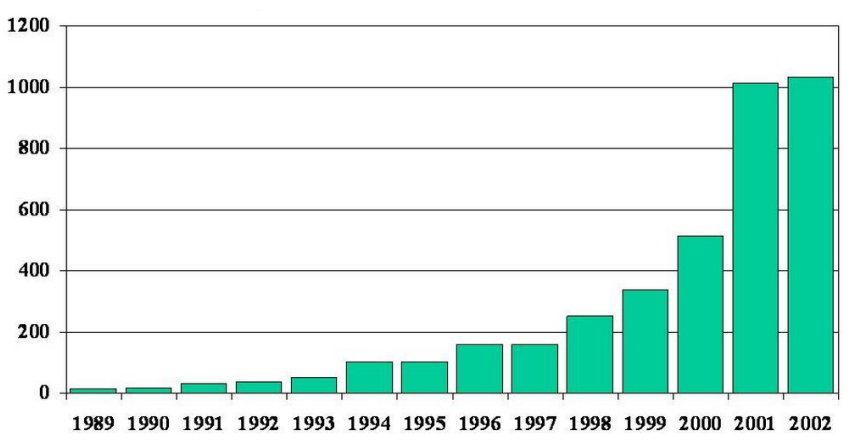

Figura 1. Numero de casos de Encefalopatia Espongiforme Bovina (EEB) reportados mundialmente, 1989-2002.

\section{Comentarios Finales}

El reto que enfrentan los países miembros es cómo balancear sus necesidades regulatorias particulares contra la meta general de un comercio libre (disfrutar de los beneficios que provee el libre comercio y al mismo tiempo minimizar el riesgo de introdución y propagacion de pestes, enfermedades y malezas). Un acuerdo mundial de MSF es una ayuda pero no una panacea. El motivo principal de las 
MSF es facilitar el comercio. Sin embargo, necesitamos asegurarnos de que los beneficios del comercio se mantengan estables. La importación de organismos dañinos pueden fácilmente borrar estas ganacias. Se debe recordar que la primera línea de defensa de un país es la prevención y que la prevención es siempre menos costosa que la erradicación.

\section{Referencias}

Food and Agricultural Organization (FAO). 2001. The state of food and agriculture 2001. Rome, Italy: FAO.

http://www.fao.org/docrep/003/x9800e/x9800e14.htm

James, S., and K. Anderson. 1998. On the Need for More Economics Assessment of Quarantine Policies. Australian Journal of Agricultural and Resource Economics 42(4):425-444.

McNeely, J.A. 1999. An Introduction to Human Dimensions of Invasive Alien Species. In The Great Reshuffling: Human Dimensions of Alien Invasive Species, edited by J.A. McNeely, pp. 5 22. Gland, Switzerland: IUCN Publishers.

Roberts, D. 1998. Implementation of the WTO Agreement on the Application of Sanitary and Phytosanitary Measures. Agriculture in the WTO/WRS-98-44. Economic Research Service/ USDA, Washington, D.C.

USDA Briefing Room. 2003. http://www.ers.usda.gov/briefing/invasivespecies/ indemnities.htm.

USDA. 2003. Floriculture and Nursery Crops Situation and Outlook Yearbook. FLO-2003. Market and Trade Economics Division, Economic Research Service, USDA, Washington, D.C. (June).

World Trade Organization (WTO). 1995. Agreement on the Application of Sanitary and Phytosanitary Measures. In Results of the Uruguay Round of Multilateral Trade Negotiations: The Legal Texts. Geneva, Switzerland: World Trade Organization.
World Trade Organization website http://www.wto.org/english/thewto_e/whatis_e/ inbrief_e/inbr00_e.htm. 